\title{
Study on the hyperspectral polarized reflection characteristics of oil slicks on sea surfaces
}

\author{
SUN ZhongQiu*, ZHAO YunSheng*, YAN GuoQian \& LI ShaoPing \\ College of Urban and Environmental Sciences, Northeast Normal University, Changchun 130024, China
}

Received March 8, 2010; accepted July 6, 2010; published online April 13, 2011

\begin{abstract}
This study is concerned with the need for remote sensing techniques for the monitoring of oil-slick pollution on sea surfaces and the effects of oil-slick pollution on the sea. We used Daqing crude oil, Jilin crude oil, heavy oil and seawater from Dalian Bay to simulate oil-slick pollution on the sea surface and obtained multi-angle hyperspectral polarized reflectance information, from which we calculated the polarization for different kinds of crude oil, and oil-slicks of different thicknesses. By comparing the degree of polarization between oil-slicks of different thickness, and sea water, it was found that in the case of thin oil-slicks, the degree of polarization of seawater is higher than that of oil slicks with wavelengths of between $400-1000 \mathrm{~nm}$. However, there was little difference at a wavelength of $785 \mathrm{~nm}$. At angles of incident of $20^{\circ}$ and $30^{\circ}$ (the viewing angle equals the incident angle), it was easy to distinguish the changes of oil-slick thickness by the degree of polarization at 785 and $880 \mathrm{~nm}$ in the near-infrared band. The crude oil showed its inherent polarized characteristic as the oil-slick thickness increased to a certain degree. That is to say, the polarization of the seawater was higher than that of the oil-slick in the visible light range, but less than that in the near-infrared band. As the incident angle changed from $40^{\circ}$ to $50^{\circ}$, the degree of polarization of seawater increased, and it was higher than that for the oil-sick between wavelengths of $400-1000 \mathrm{~nm}$ at an angle of incidence of $50^{\circ}$. This research on the polarized characteristics of an oil-slick on a sea surface brings new scientific techniques to the monitoring of sea-surface pollution by remote sensing.
\end{abstract}

remote sensing, hyperspectral, oil-slick pollution, degree of polarization

Citation: Sun Z Q, Zhao Y S, Yan G Q, et al. Study on the hyperspectral polarized reflection characteristics of oil slicks on sea surfaces. Chinese Sci Bull, 2011, 56: 1596-1602, doi: 10.1007/s11434-010-4112-5

Oil from offshore spills is one of the common marine pollutants; it causes serious pollution of the marine environment and has harmful effects on marine life. Oil spills on the sea surface can obstruct the ocean-atmosphere heat exchange and influence the absorption, transmission and reflection of light waves by the sea surface [1]. When vast areas of the sea surface have been polluted, we can use remote sensing technology to do a real time, high speed, macro monitoring of the polluted regions. Studies on the condition and process of oil spills on sea surfaces are the subject of much attention worldwide [2-4].

With the rapid development of remote sensing technology, researchers are focusing on using hyperspectral remote-

*Corresponding authors (email: sunzq465@nenu.edu.cn; zhaoys975@163.com) sensing to monitor the oil spills on sea surfaces $[5,6]$. However, in general, their research is usually concerned with normal observations of the surface which gather the vertical information of objects on the surface [7]. Palmer pointed out that marine oil spills have different reflectance characteristics for wavelengths of 700, 740 and $800 \mathrm{~nm}$ when the oil spill concentration changes. The most suitable band for extracting oil slick information is 440-900 nm [8]. Foudan showed that the band of $600-900 \mathrm{~nm}$ has the greatest remote sensing detection potential for oil slicks [5]. Zhao summarized the changes in spectral characteristic with changes in thickness of diesel oil, lubricating oil and crude oil slicks [6]. Zhang et al. analyzed the change in marine oil spill spectral characteristics, and the results showed that 500-580 $\mathrm{nm}$ provides the maximum reflectance for oil 
slicks [1]. Recently, Lu et al.'s marine oil slick experiments indicated that different oil thicknesses resulted in varied oil slick spectral characteristics and responses. At the same time, research on thin oil slicks on a sea surface showed that the light incident on oil slicks accreted onto the sea surface was subjected to visible/near-infrared reflectance because of its multiple reflection and refraction $[9,10]$. Wettle et al. carried out quantitative analysis on oil slicks of different thickness and different kinds of oil, combining these with the measured spectra and images [11]. The studies above showed that research on oil slicks on the sea surface is the main focus for marine oil spill remote sensing technology. The issue is, are there any other remote sensing techniques that have advantages in oil slick thickness monitoring?

When using hyperspectral remote sensing data to improve the ability of remote sensing to recognize surface features, people noticed the influence and contribution of information on angle and polarization in object recognition. Compared with traditional methods, multi-angle earth observation could obtain more specific and reliable three-dimensional structural parameters of a ground target. This would meet the requirement of a quantitative remote sensing inverting parameter, which would make quantitative remote sensing possible [12]. In recent research on polarized remote sensing [13-15], the importance of polarized information in recognizing surface features has been recognized. One of the best examples of the method of combining multi-angle viewing with polarization was provided by the airborne POLDER instrument on the CNES satellite PARASOL. The instrument gathered polarized and directive data on the solar radiation reflectance of the earth-atmosphere system from space by global observation $[16,17]$.

To establish the reflectance characteristics of oil slicks in more detail, Talmage et al. [18] measured multi-angle polarized reflection to calculate polarization. Zhao et al. has also done quantitative research on the multi-angle polarized reflection and bidirectional reflection of oil spills on water [7]. However, research on oil thickness and oil types combining hyperspectral information and multi-angle polarization is still at an early stage. Therefore, we need to take the next step of exploring the multi-angle hyperspectral polarized response to drift, diffusion, division, evaporation and other processes with different kinds of oil poured into marine environments, to provide the technology to correctly identify the type of marine oil, the thickness of the oil slick and even the amount of oil $[19,20]$. Compared with non-polarization measurements, polarization measurements can gather information from which it is easier to identify the characteristics of a target from certain surface features. Polarized remote sensing, especially in dark environments, has greater potential and advantages in recognizing dark objects with low reflectance. It provides a new effective method of remote sensing for water bodies [21]. Noting the advantage of polarized remote sensing in determining low reflectance, this study used polarization as the measurement index, com- bining it with hyperspectral information to analyze the oilpolluted sea surface from different angles, and obtain the polarization of different kinds of oil-polluted sea surfaces and the thicknesses of oil slicks at wavelengths of 400-1000 $\mathrm{nm}$. This study also discusses the correlation between polarization and oil slick thickness, providing a useful method of using remote sensing technology to monitor marine oil slick thickness and oil grades.

\section{The theoretical basis of polarization technology}

Polarization is a basic characteristic of vector waves. The phenomenon, in a narrow sense, is characterized by light waves with different amplitudes for different incident directions in the plane vertical to the direction of propagation. When light irradiates the sea surface at an angle, reflection and refraction occur. Ideally, we divide incident light into a component parallel to the incident plane called $P$, and a component perpendicular to the incident plane called $S$, then according to Fresnel reflectance equation:

$$
\begin{gathered}
r_{p}=\frac{E_{1 p}^{\prime}}{E_{1 p}}=\frac{\tan \left(i_{1}-i_{2}\right)}{\tan \left(i_{1}+i_{2}\right)}, \\
r_{s}=\frac{E_{1 s}^{\prime}}{E_{1 s}}=\frac{\sin \left(i_{2}-i_{1}\right)}{\sin \left(i_{2}+i_{1}\right)} .
\end{gathered}
$$

As the sensor receives the reflectance light intensity from the sea surface, we focus in this study on the reflection equation. $E_{1}$ and $E_{1}^{\prime}$ are light vectors of the incident and reflected light, $i_{1}$ and $i_{2}$ are the incident and refracted angles, $r_{p}$ and $r_{s}$ are the amplitude reflectance of components $P$ and $S$. Ignoring the directions of the light, from eqs. (1) and (2):

$$
r_{p}=r_{s} \cdot \frac{\left|\cos \left(i_{1}+i_{2}\right)\right|}{\cos \left(i_{1}-i_{2}\right)} .
$$

When the incident light is perpendicular to the sea surface, $i_{1}=0^{\circ}, r_{s}=r_{p}$, so the reflected light has no polarization. When $0^{\circ}<i_{1}<90^{\circ}$,

$$
\begin{gathered}
\left|\cos \left(i_{1}+i_{2}\right)\right|<\cos \left(i_{1}-i_{2}\right), \\
r_{p}<r_{s} .
\end{gathered}
$$

Eq. (5) shows that the value of the parallel component of the electric vector in reflected light is always less than the vertical one, so when the incident light is natural light, its reflection shows polarization.

The state of polarization of a light beam can be fully described as four Stokes Parameters, defined as

$$
\left\{\begin{array}{l}
I=I_{0^{\circ}}+I_{90^{\circ}}=I_{+45^{\circ}}+I_{-45^{\circ}}, \\
Q=I_{0^{\circ}}-I_{90^{\circ}}, \\
U=I_{+45^{\circ}}-I_{-45^{\circ}}, \\
V=I_{r}-I_{l},
\end{array}\right.
$$


$I_{0^{\circ}}, I_{90^{\circ}}, I_{445^{\circ}}, I_{-45^{\circ}}, I_{l}$, and $I_{r}$ are the light intensity of line polarized light, left $(l)$ circularly polarized and right $(r)$ circularly polarized light at $0^{\circ}, 90^{\circ},+45^{\circ}$, and $-45^{\circ}$ directions of an ideal polarizer which is placed in the light transmission path. Polarization is defined as the ratio between the intensity of the polarization component and the total intensity of the light:

$$
P=\frac{\sqrt{Q^{2}+U^{2}+V^{2}}}{I} .
$$

Because of the circular polarization, components $(V)$ of most surface features are weak and neglected [22]. So polarization can be expressed as

$$
P=\frac{\sqrt{Q^{2}+U^{2}}}{I}=\frac{\sqrt{\left(I_{0^{\circ}}-I_{90^{\circ}}\right)^{2}+\left(I_{+45^{\circ}}-I_{-45^{\circ}}\right)^{2}}}{I_{0^{\circ}}+I_{90^{\circ}}} .
$$

When natural light irradiates a smooth liquid surface with a non-Brewster angle, both the reflected and refracted light changes into partially polarized light, and the main vibration direction of the reflected light must be vertical to the incident interface, while the main vibration direction of the refracted light is in the incident plane, so parameter $Q$ is negative. When the incident angle gradually increases, polarization increases as well, and when it reaches the Brewster angle, the polarization decreases. To calculate the polarization of marine oil slicks accurately, the simulated liquid level in this experiment is initially at rest. Taking into account the measurement of polarized light at $0^{\circ}, 90^{\circ},+45^{\circ}$, and $-45^{\circ}$, we calculated their polarization by eq. (8).

\section{Materials and methods}

\subsection{Experimental environment and equipment}

The experiment was carried out in a dark room using the bidirectional reflectance measuring equipment developed by Changchun Optical Machine Institute, Chinese Academy of Sciences, and the USB2000 spectrometer made by Ocean Optics Company, to measure the reflected spectrum. The bi-directional reflectance photometer used a nickel-tungsten lamp as the light source, which could be set at every $10^{\circ}$ as a light source with an incident zenith angle in the range of $0^{\circ}-70^{\circ}$. There were seven detecting heads on the detection frame, spaced $10^{\circ}$ from each other, on the range of $0^{\circ}-70^{\circ}$. There was a detection position every $10^{\circ}$ from $0^{\circ}-360^{\circ}$ between the light source and the detector $\left(180^{\circ}\right.$ was the specular reflection direction). The equipment also had a polarized lens, which could rotate to any angle. The detection wavelength range of the hyperspectral light source was $340-1050 \mathrm{~nm}$. The viewing angle of the detection optical fiber was $25^{\circ}$. The integration time was between 3-65 ms. The data transfer rate refreshed the memory each $13 \mathrm{~ms}$. When starting the USB2000 Spectrometer, it reads the parameters of the wavelength automatically using the OOIbase32TM operating software.

\subsection{Experimental materials}

Materials used in this experiment were Daqing crude oil, Jilin crude oil and heavy oil. The density of Jilin crude oil is $0.859 \mathrm{~g} / \mathrm{cm}^{3}$, and it has a low freezing point, low wax content, poor viscosity, and a brownish-black appearance. The density of heavy oil is $0.946 \mathrm{~g} / \mathrm{cm}^{3}$, and it is more viscous, has low volatility, and a black appearance. There were two samples of Daqing crude oil, named Daqing No.1 and Daqing No.2. The density of Daqing No.1 is $0.895 \mathrm{~g} / \mathrm{cm}^{3}$, and it has a brown appearance, a high freezing point and high wax content. The density of Daqing No.2 is 0.884 $\mathrm{g} / \mathrm{cm}^{3}$, and it has a brownish-black appearance, a high freezing point and high wax content. The seawater in this experiment was from Dalian Bay, with a density of 1.02165 $\mathrm{g} / \mathrm{cm}^{3}$, and clear water quality.

\subsection{Experimental methods}

In this study, we put the seawater samples into containers wrapped with black tape, to prevent the specular reflection of incident light at the side wall and bottom and eliminate measurement errors. During the measurement, the distance between the spectrometer probe and the oil slick surface was $5 \mathrm{~cm}$, and the detection region was $3.8 \mathrm{~cm}^{2}$, which was smaller than the container's diameter. To avoid the influence of oil slick spectral reflection from the container's bottom, we made a special container of diameter $8 \mathrm{~cm}$ and depth $24 \mathrm{~cm}$. We dropped crude oil into the seawater, and then waited for the oil slick to diffuse and fill the container port.

To make the status of the oil slick easily observable, we used a transparent glass as shown in Figure 1. Because the crude oil and heavy oil were quite thick, we melted the oil in $50^{\circ} \mathrm{C}$ water before dropping it into seawater. Using a glass burette and plastic head balloon several times, the results showed that $1 \mathrm{~mL}$ of crude oil and heavy oil both contained 20 drops, and the thickness of the oil slick was about $400 \mu \mathrm{m}$ using the container diameter to calculate the surface area. We used a spectrometer to measure the polarization of crude oil, heavy oil, seawater and oil-polluted seawater with different slick thicknesses at polarizing angles of $0^{\circ}, 90^{\circ}$, $+45^{\circ}$, and $-45^{\circ}$, when the azimuth angle was $180^{\circ}$ and the incident angle equaled the search angles at $20^{\circ}, 30^{\circ}, 40^{\circ}$, and $50^{\circ}$. We then calculated the polarization using eq. (8). To maintain the precision of this study, we not only ensured the incident angle equaled the search angle, but also ensured the same liquid level by using a $2 \mathrm{~mL}$ burette. We repeated the experiments six times to get the arithmetic mean values for each polarization.

\section{Results and analysis}

In this study, we chose a spectrum of between $400-1000 \mathrm{~nm}$ 


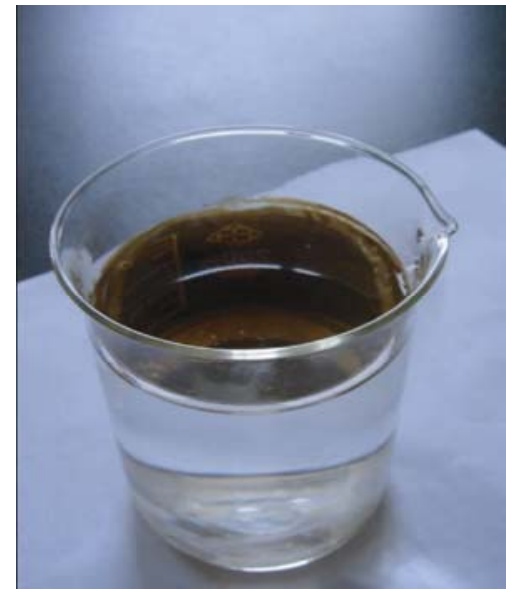

Figure 1 Crude oil slick on the sea surface.

because of noise. We call the incident and search zenith angles, the incident and search angles for short, and the incident angle equals the search angle.

\subsection{Analysis of the polarization of seawater, crude oil and heavy oil changes with light wavelength band}

Figure 2 shows that when the incident angle is $20^{\circ}, 30^{\circ}, 40^{\circ}$, and $50^{\circ}$, the curve of the polarization of seawater, crude oil and heavy oil is between $400-1000 \mathrm{~nm}$. In this figure, the polarization from bottom to top is given for incident angles $20^{\circ}, 30^{\circ}, 40^{\circ}$, and $50^{\circ}$, in that order. As can be seen, the polarization of the three crude oil samples and the heavy oil increased as the light wavelength band increased, and the rate of increase in the near-infrared band is faster than in the visible band. All of the curves showed two troughs at 785 $\mathrm{nm}$ and $880 \mathrm{~nm}$. Comparing the curve of polarization of crude oil and heavy oil at the same incident angle, we see that the polarization in descending order is heavy oil, Jilin crude oil, Daqing No.2 crude oil and Daqing No.1 crude oil. Daqing No.1 and Daqin No.2 show little difference in polarization in the near-infrared band but greater difference in the visible band, which is related to their differing colors.

According to our analysis of seawater, crude oil and

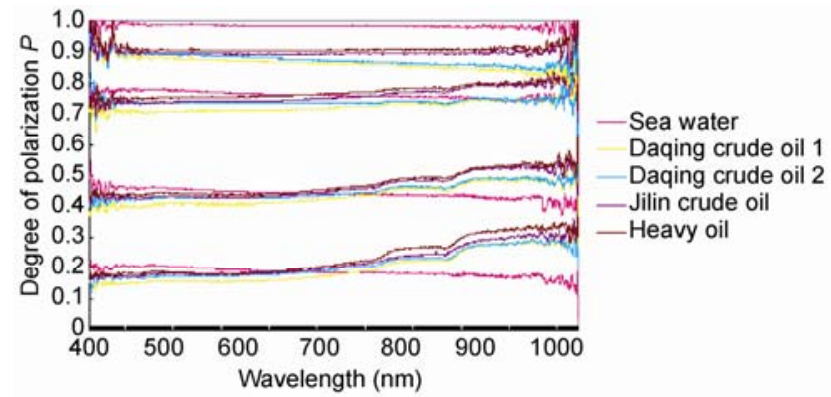

Figure 2 Curves of hyperspectral polarization of seawater, crude oil and heavy oil, with the incident angle equal to the search angle at $20^{\circ}, 30^{\circ}, 40^{\circ}$, and $50^{\circ}$, and the azimuth angle at $180^{\circ}$. heavy oil, we found that the two troughs at $785 \mathrm{~nm}$ and 880 $\mathrm{nm}$ in the near-infrared band are inherent properties of oil. When the incident angle was $50^{\circ}$, the seawater was nearly at the Brewster angle; therefore, the polarization corresponding to the bands showed no significant differences. Because crude oil and heavy oil absorb liquid, their polarization reaches a maximum value that is, however, less than 1 [23].

\subsection{Analysis of polarization of different thicknesses of the oil slick on the sea surface}

In the simulation experiments of the sea surface slick pollution, we compared the polarization of different oil slick thicknesses to describe the influence of polarization by oil slick thickness. Because the freezing points of Daqing crude oil and heavy oil are higher than that of Jilin crude oil, it is quite difficult to make oil slicks distribute evenly on the sea surface. Therefore, when dropping heavy oil and Daqing crude oil into seawater, we increased the oil to broaden the sea area coned by the oil slicks, and to attain a thickness of $40 \mu \mathrm{m}$. However, we could only attain a thickness of $20 \mu \mathrm{m}$ with Jilin crude oil.

Figure 3 shows the curve of polarization of seawater and different polluted sea surfaces with changes in the light wavelength band. While undertaking this experiment, we found that the changes in polarization of Daqing No.1 and No.2 crude oil with equivalent numbers of drops are the same as crude oil. That is to say, there were differences in the visible band but few in the near-infrared band. To show the polarization of sea surfaces polluted by different kinds of oil clearly, Daqing No.2 crude has not been presented. The polarizations of the oil slicks were all lower than that of seawater, but showed only small differences at $785 \mathrm{~nm}$.

With the aim of describing the effect of a change in oil slick thickness on polarization, we chose Jilin crude oil, which is easy to control, with thicknesses of 20,60,160 and $220 \mu \mathrm{m}$. It can be seen from Figure 4 that when the incident angle changes from $20^{\circ}$ to $30^{\circ}$, and $40^{\circ}$, and as the thickness increases, the polarization also increases. In addition, the change of polarization in the near-infrared band is clearer than in the visible band. This is because the absorption rate

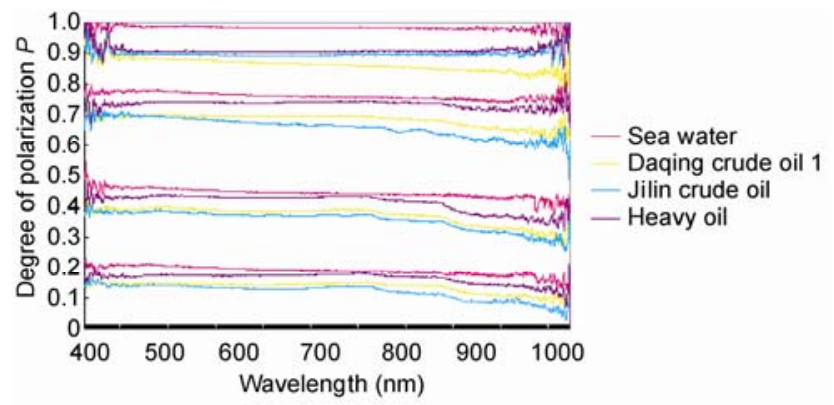

Figure 3 Curve of hyperspectral polarization of seawater and different oil slicks, with incident angles of $20^{\circ}, 30^{\circ}, 40^{\circ}$, and $50^{\circ}$, and an azimuth angle at $180^{\circ}$. 


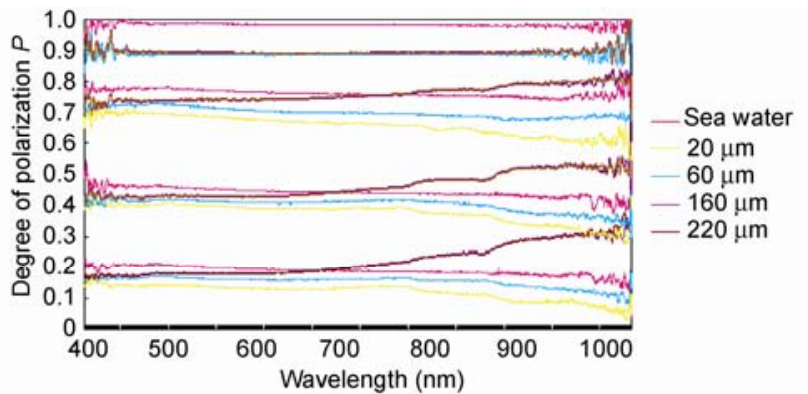

Figure 4 Curves of hyperspectral polarization of seawater and different oil slicks, with incident angles of $20^{\circ}, 30^{\circ}, 40^{\circ}$, and $50^{\circ}$, and an azimuth angle at $180^{\circ}$.

of crude oil in the near-infrared band is higher than in the visible band. With the increase in thickness, as shown in Figure 5, the initial refracted light into the crude oil slick was mainly absorbed, while the thicker the slick was, the more it could absorb, and the less light reflected on the oil and air interface, so its polarization was closer to that of crude oil. In the process of the oil slick thickening from the initial thin state, the polarization values at $785 \mathrm{~nm}$ and 880 $\mathrm{nm}$ changed from peaks to troughs. This is primarily because the change in oil thickness resulted in a change in refracted light $a_{2}$, which caused the changes at $785 \mathrm{~nm}$ and $880 \mathrm{~nm}$. We found the same phenomenon while researching the effect of polarization with changes of thickness in oil slicks for both Daqing crude oil and heavy oil, so we will not discuss it here.

\subsection{Analysis of polarization of oil slick with different incident angles}

It can be seen in Figures 2-4 that as the incident angle increases, the polarization of seawater, crude oil, heavy oil and oil slicks with different thicknesses also increases. When the incident angle is $20^{\circ}$, the polarization of seawater below $630 \mathrm{~nm}$ is higher than for heavy oil and similar kinds of crude oil, but less than for those above $775 \mathrm{~nm}$. When the incident angle is $30^{\circ}$, the polarization of seawater below 650 $\mathrm{nm}$ is higher than for heavy oil and similar kinds of crude

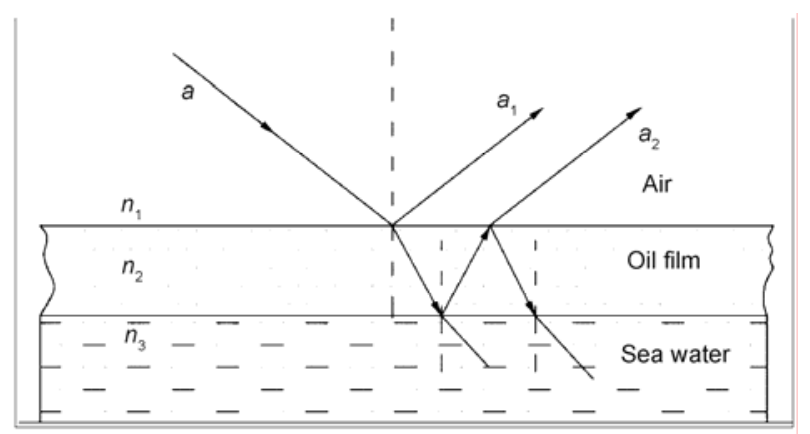

Figure 5 Changes of reflection and refraction of the light incident onto the oil slick surface. oil, but lower than for those above $800 \mathrm{~nm}$. However, when the incident angle is $40^{\circ}$, the band where the polarization of seawater is higher than the others becomes wider. As the incident angle increases, the two troughs at $785 \mathrm{~nm}$ and $880 \mathrm{~nm}$ are gradually smoothed out, and tend to be a line when the incident angle reaches $50^{\circ}$. The polarization of seawater decreases while the band increases, and has no significant fluctuations. When the incident angle is $50^{\circ}$, the polarization of seawater is higher than those of crude oil and heavy oil on the whole range of the band. This is because, as the incident angle increases, the changing speed of the vertical and parallel components of crude oil and heavy oil is slower than for seawater.

From Figure 3, we can see that, as the incident angle increases, the polarizations of different oil slicks on the sea surface would increase, and the polarizations of oil slicks are all less than for sea water, but have little difference at about $785 \mathrm{~nm}$. This is because the light incident to the slick surface, as in Figure 5, divides into two parts, one is the reflected light $a_{1}$, and the other part has been refracted into the oil slick, and then meets the second plane, where it is again divided into reflected and refracted light. The reflected light passes through the oil-air interface as the refracted light $a_{2}$. In fact, the beam is reflected many times in the oil slick, then refracts from the oil slick surface into the air, but these light intensities are all weaker than $a_{1}$ and $a_{2}$, do little in superposition, and can be ignored [24]. With regard to the dominant reflected light amplitude component perpendicular to the incident plane and the dominant refracted light amplitude component parallel to the incident plane, the refracted light $a_{2}$ is produced by a double refraction, so the refracted light amplitude component parallel to the incident plane dominates, which increases the $Q$ parameter. The $Q$ parameter is always negative, so $Q^{2}$ decreases, but the $I$ parameter increases, and the polarization calculated by eq. (8) also decreases. Therefore, when there is an oil slick on the sea surface, the level of polarization is less than the polarization of seawater. When the incident angle increases, because of the refraction of the oil slick, $\left(n_{2}\right)>$ seawater $\left(n_{3}\right)>$ air $\left(n_{1}\right)$, total reflection would occur in the oil slick. That is to say, the polarization detected should be the same as the polarization of crude oil and heavy oil. When the incident angle is $50^{\circ}$, the polarization of the oil slick as shown in Figure 3 is generally the same as the polarization of crude oil and heavy oil as shown in Figure 2. This indicated that total reflection happened on the oil-air interface.

In addition, when the incident angles are $20^{\circ}, 30^{\circ}$, and $40^{\circ}$, grouped according to the type of oil slick, the polarizations, in descending order, are heavy oil, Daqing crude oil, and Jilin oil. This is because the thickness of heavy oil and Daqing crude oil is greater than that of Jilin oil, which can absorb much more refracted light and reduces the second-refracted light component $a_{2}$, so their polarization is higher than that of Jilin crude oil. However, the two troughs 
at $785 \mathrm{~nm}$ and $880 \mathrm{~nm}$ have turned into peaks, and as the incident angle increases, the peaks gradually smooth out.

While treating Jilin crude oil as the major study subject, we found that as the thickness of the oil slick increases, its polarization decreases, while the incident angle increases from $20^{\circ}$ to $30^{\circ}$ and then $40^{\circ}$. This is because the increase in thickness increases the optical path, and thus increases the energy absorption, which results in a decrease in the second refracted intensity $a_{2}$. When the incident angle is $50^{\circ}$, the polarizations of oil slicks of different thicknesses are nearly the same. Irrespective of how large the incident angle is, the polarization of the oil slick with a thickness of $160 \mu \mathrm{m}$ is nearly the same as that of crude oil. In other words, when the slick thickness increases to a certain extent, the refracted light $a_{2}$ can be ignored and the internal reflection nearly disappears.

\section{Conclusions and discussion}

When the incident angle equals the search angle in the range $20^{\circ}-50^{\circ}$, with the azimuth angle at $180^{\circ}$, as the incident angle increases, the polarizations of sea surface, crude oil, heavy oil and oil-polluted sea surface also increase. Different types of crude oil have different polarizations, but they all have two troughs at 785 and $880 \mathrm{~nm}$. The two demarcation points of the polarization of seawater occur at 630 and $775 \mathrm{~nm}$, the polarization of seawater below $630 \mathrm{~nm}$ is higher than for heavy oil and similar kinds of crude oil, but it is less than these above $775 \mathrm{~nm}$. However, with an increase of the incident angle, the polarization of seawater in the near-infrared band range becomes higher than that for crude and heavy oil. When the incident angle is $50^{\circ}$, its polarization is higher than that for crude oil on the whole light band range of wavelengths.

Because of the very nature of oil slicks, the polarization of different kinds of oil slick polluted seawater present differences. When the oil slick is thin, its polarization is less than that of seawater, but there is little difference at $785 \mathrm{~nm}$. A common feature of the polarization of crude oil and heavy oil is that two troughs at 785 and $880 \mathrm{~nm}$ turn into peaks. As the thickness of the oil slick increases, the polarization increases, and the rate of increase in the near-infrared band is faster than that in the visible band. However, when the incident angle is $50^{\circ}$, the change in the thickness of an oil slick has no effect on polarization, so it is not advisable to choose a larger incident angle when distinguishing oil slick thicknesses.

According to the analysis of the multi-angle hyperspectral curve of seawater and an oil slick polluted sea surface, we know that when the incident angle is $20^{\circ}$ and $30^{\circ}$, it is easy to distinguish between seawater and oil-polluted water by using the near-infrared band. We can also determine the thickness of an oil slick by its polarization at 785 and $880 \mathrm{~nm}$.
In conclusion, the use of multi-angle hyperspectral polarized remote sensing technology to monitor the pollution area of a marine oil slick, the type of oil slick pollution and thickness of the oil, shows great promise and significant potential for application in the future.

This work was supported by the National Basic Research Program of China (2009CB426305), National Natural Science Foundation of China (40971190, 40771153), Nurturing Project of Scientific and Technological Innovation Platform, Northeast Normal University (106111065202).

1 Zhang Y N, Ding Q, Gao C, et al. Analysis of oil film spectrum and monitoring oil spilled by remote sensing. Mar Environ Sci, 2000, 19: $5-10$

2 O'Brien G W, Lawrence G M, Williams A K, et al. Yampi Shelf, Browse Basin, North-West Shelf, Australia: A test-bed for constraining hydrocarbon migration and seepages rates using combinations of 2D and 3D seismic data and multiple independent remote sensing technologies. Mar Petrol Geol, 2005, 22: 517-549

3 Brown C E, Fingas M F. Development of airborne oil thickness measurement. Mar Pollut Bull, 2003, 47: 485-492

4 Keramitsoglou I, Cartalis C, Kiranoudis C T. Automatic identification of oil spills on satellite images. Environ Modell Softw, 2006, 21: 640-652

5 Salem F M F. Hyperspectral remote sensing: A new approach for oil spill detection and analysis. Doctoral Dissertation. Washingtm D C: George Mason University, 2003. 49-85

6 Zhao D Z, Cong P F. The research of visual light wave-band feature spectrum of sea-surface oil spill. Remote Sens Techn Appl, 2000, 15: $160-164$

7 Zhao Y S, Wu T X, Luo Y J, et al. Research on quantitative relation between polarized birdirectional reflectance and bidirectional reflectance of water-surface oil spills. J Remote Sens, 2006, 10: 294-298

8 Palmer D, Boasted G A, Boxall S R. Airborne multispectral remote sensing of the January 1933 Shetlands oil spill. In: Proceedings of the second Thematic Conference on remote sensing for Marine and Coastal Environments: Needs, Solutions and Applications, ERIM Conferences, Ann Arbor, 1994, 11: 545-558

9 Lu Y C, Tian Q J, Wang J J, et al. Spectral response analysis of oil slicks. Chinese Sci Bull, 2008, 53: 1085-1088

10 Lu Y C, Tian Q J, Qi X P, et al. Spectral response analysis of offshore thin oil slicks. Spectrosc Spectr Analys, 2009, 29: 986-989

11 Wettle M, Daniel P J, Logan G A, et al. Assessing the effect of hydrocarbon oil type and thickness of a remote sensing signal: A sensitivity study based on the optical properties of two different oil types and the HUMAP and Quickbird sensors. Remote Sens Environ, 2009, 113: 2000-2010

12 Henderson B G, Theiler J, Villeneuve P. The polarized emissivity of a wind-roughened sea surface: A Monte Carlo model. Remote Sens Environ, 2003, 88: 453-467

13 Zhang C Y, Cheng H F, Chen Z H, et al. Detecting low reflectivity camouflage net by using polarization remote sensing. J Inf Mill Wav, 2009, 28: 137-140

14 Wu T X, Yan L, Xiang Y, et al. Polarization reflection effect of plane rough surface under vertical observation. J Inf Mill Wav, 2009, 28: $151-155$

15 Wang Y, Sheng L X, Li K, et al. Measurement of light polarization characteristics from an oil-polluted soil surface in near-infrared bands. Chinese Sci Bull, 2008, 53: 2956-2961

16 Csiszar I, Gutman G, Romanov P, et al. Using ADEOS/POLDER data to reduce angular variability of NOAA/AVHRR reflectances. Remote Sens Environ, 2001, 76: 399-409

17 Leroy M, Breon F M. Angular signatures of surface reflectances from airborne POLDER data. Remote Sens Environ, 1996, 57: 97-98 
18 Talmage D A, Curran P J. Remote sensing using partially polarized light. Int J Remote Sens, 1986, 7: 47-64

19 Brekke C, Solbreg A H S. Oil spill detection by satellite remote sensing. Remote Sens Environ, 2005, 95: 1-13

20 Gade M, Alpers W. Using ERS22 SAR Images for routine observation of marine pollution in European Coastal Waters. Sci Total Environ, 1999, 237-238; 441-448

21 Egan W G, Duggin M J. Comparative merits of multispectral optical polarization to microwave. Proc SPED, 2002, 4481: 292-298

22 Sun X B, Qiao Y L, Hong J, et al. Experimental Study on polarization characteristic of man-made objects. High Technol Lett, 2003, 8: 23-27

23 Wang S. Crystal Optics of the Opaque Minerals (in Chinese). Beijing: Geological Publishing House, 1987. 35-39

24 Yao Q J. Optical Study Course (in Chinese). Beijing: Higher Education Press, 2008. 30-36

Open Access This article is distributed under the terms of the Creative Commons Attribution License which permits any use, distribution, and reproduction in any medium, provided the original author(s) and source are credited. 